\title{
Understanding behavior makes it more normal
}

\author{
WOO-KYOUNG AHN and LAURA R. NOVICK \\ Vanderbilt University, Nashville, Tennessee \\ and \\ NANCY S. KIM \\ Yale University, New Haven, Connecticut
}

\begin{abstract}
Meehl (1973) has informally observed that clinicians will perceive a patient as being more normal if they can understand the patient's behaviors. In Experiment 1, undergraduate participants received descriptions of 10 people, each with three characteristics(e.g., frequently suffers from insomnia) taken from the Diagnostic and Statistical Manual of Mental Disorders (American Psychiatric Association, 1994). When the characteristicsformed a plausible causal chain, adding a causal explanation increased perceived normality; but when a causal chain was implausible, perceived normality decreased. In Experiments 2 and 3, a negative life event (e.g., is very stressed out due to her workload) was added as an explanation for the first characteristic in a three-characteristiccausal chain. Undergraduates, graduate students in clinical psychology, and expert clinicians all reliably perceived the patients as being more normal with these explanations than without them, confirming Meehl's prediction.
\end{abstract}

Imagine a student who forgets the names of her family members or a taxi driver who says that others listen to his thoughts. Most people's immediate reaction would be that there is something wrong with these people; they are abnormal. These examples illustrate that not only professionals, such as clinical psychologists and psychiatrists, but also lay people in everyday situations are constantly judging (with or without awareness) others' behavior and psychological health as normal or abnormal. What affects people's judgments of the normality of other people?

Our work on this issue was inspired by an informal observation made by Meehl (1973). He noted that when clinicians felt that they understood a patient, the patient seemed normal; that is, "understanding it makes it normal" (p. 244). Meehl identified this as a fallacy in clinical reasoning, arguing that understanding why a man deliberately killed his wife does not make the act a normal, excusable one. The goal of the present research was to provide the first empirical demonstration that understanding the cause(s) of a person's behavior makes that person appear more normal.

In previous studies, the consequences of causal explanations or attributions in other domains have been examined. It has been found that explanations affect memory (e.g., Crocker, Hannah, \& Weber, 1983), decision mak-

This project was supported by National Institute of Mental Health Grant RO1 MH57737, awarded to the first author, and by a National Science Foundation Graduate Research Fellowship to the third author. We thank Jessecae Marsh for collecting the data and Patti van Eys for help in the selection of stimuli. Correspondence concerning this article should be sent to W.-K. Ahn, Department of Psychology, Yale University, P. O. Box 208205, New Haven, CT 06520-8205 (e-mail: woo-kyoung. ahn@yale.edu). ing (e.g., Carroll \& Payne, 1977; Pennington \& Hastie, 1992; Shafir, Simonson, \& Tversky, 1993), problem solving (e.g., Chi, Bassok, Lewis, Reimann, \& Glaser, 1989), affective responses (e.g., Moore, Strube, \& Lacks, 1984), probability judgments (e.g., Koehler, 1991), and categorization (e.g., Ahn, Brewer, \& Mooney, 1992).

The most relevant finding among these studies is the so-called explanation effect in probability judgments: People who explain a possibility later believe that the possibility is more likely to occur (see Koehler, 1991, for a review). For instance, after explaining a hypothetical victory by a randomly chosen team in a college football game, participants believed that their chosen team was more likely to win (Sherman, Zehner, Johnson, \& Hirt, 1983). These findings suggest that providing explanations for abnormal behavior might make the behavior look more normal by making it appear more likely to occur or more prevalent. Kahneman and Tversky's (1982) simulation heuristic makes the same prediction. They proposed that people believe that the more easily a causal scenario can be constructed, the more likely the event should be to occur.

In the present experiments, participants received descriptions of people, each with three characteristics taken from the DSM-IV (American Psychiatric Association, 1994), and were asked to judge the normality of these people. The experiments manipulated the comprehensibility of these characteristics. Meehl (1973) did not articulate in technical terms what he meant by "understanding" a person. In common usage, people typically assume that understanding behavior involves determining its underlying cause(s). Thus, our experiments focused on the role of causal explanations. We explored two possibilities that are highly likely to occur in ordinary situations involving the perception of persons. 
First, people often attempt to explain how observed behaviors are causally interconnected. For instance, after observing that Joan is popular, is rich, and works very hard, one might construct a story that Joan is popular because she is rich and that she is rich because she works very hard. Thagard's (1989) model for evaluating the coherence of explanations highlights the importance of such causal connections. This model is based on several principles, one of which is that an explanatory hypothesis is more coherent, the more facts it can explain. Indeed, Read and Marcus-Newhall (1993) found that a single explanation that linked several characteristics was judged to be a better explanation than were several separate explanations, one for each characteristic. In Experiment 1, we extended this work on the antecedents of explanatory coherence by investigating a possible consequence of having a coherent explanation. In particular, we examine whether merely drawing causal relations among a person's characteristics changes how normal the person is perceived to be. We predicted that when a person's characteristics were linked by plausible causal relations, the greater perceived coherence of the person's behavior would make the behavior seem more understandable, and as a result, the person would be viewed as more normal.

Second, people may attempt to discover a hidden cause underlying an observed behavior. One might wonder why Joan works so hard in the above example, or a clinician might want to uncover the cause of a patient's depressed mood. In Experiments 2 and 3, we provided an explanation for the first of the causally connected characteristics, to determine whether this additional explanation would further increase perceptions of normality. According to one of the principles in Thagard's (1989) model, explanatory hypotheses are more acceptable if they are explained by other explanatory hypotheses (see Read \& Marcus-Newhall, 1993, for empirical support). We predicted that if a set of causally connected characteristics appeared to be more coherent as a result of being further explained, the person might be viewed as more normal.

\section{EXPERIMENT 1}

In Experiment 1, we provided some participants with descriptions of how characteristics of a person were causally related to each other, whereas others received no such descriptions. The plausibility of the causal relations was manipulated to determine whether causal relations per se influence the perception of normality or whether it is the actual understanding of how characteristics fit together that makes people appear more normal. Following Meehl (1973), we predicted that understanding would be crucial.

\section{Method}

The participants ${ }^{1}$ received descriptions of 10 fictitious people, each with three mental or behavioral characteristics. For each description, they were asked to judge "how normal the person is" on a 9-point scale ( $1=$ normal, $9=$ severely disturbed $)$. Before completing this task, the participants received two examples of normal people (e.g., "Andrea who worries about her children's safety, cleans the house once a week, and is not completely satisfied with her appearance") and of severely disturbed people (e.g., "Mark who cries every day, washes his hands every hour, and wrongly believes his left arm is misshapen"), none of whom had characteristics used in the task itself. The participants were told to rate the normality of each person on the basis of their first impressions, and there were no right or wrong answers.

The participants in the causal condition $(n=33)$ learned that the characteristics in a given description formed a causal chain, whereas those in the noncausal condition $(n=38)$ were told that the characteristics did not cause each other (see Table 1 for sample materials). Each characteristic was mentioned only once in a vignette, and the order of the characteristics within a vignette was the same in the causal and the noncausal versions. In the causal condition, we manipulated the plausibility of the causal relations provided: Five items contained a plausible causal chain, and five contained an implausible causal chain. Because we deliberately selected characteristics for each item that would be causally unrelated in most people's existing background knowledge (see below), we added brief explanations for both the plausible and the implausible causal relations in the causal condition, to make them comprehensible.

The 10 descriptions were selected through pretesting from a pool of 30 items, each consisting of three features taken from the DSM-IV (American Psychiatric Association, 1994). ${ }^{2}$ Thirteen undergraduates and 1 licensed clinical psychologist rated the 30 items, described in the causal format, with respect to the plausibility of the causal relations specified among the item's characteristics, using a 10-point scale. The 5 most implausible and the 5 most plausible items were selected with the following additional constraints. First, a feature could appear only once across the 10 items. Second, the number of females and males described in the vignettes was equated across the plausible and the implausible items. Third, we excluded a set of features if it could be very easily causally related when described in the noncausal format (e.g., drinks too much caffeine and suffers from insomnia), because that would threaten the

Table 1

Sample Materials Used in the Four Conditions in Experiment 1

\begin{tabular}{lll}
\hline Condition & \multicolumn{1}{c}{ Implausible } & \multicolumn{1}{c}{ Plausible } \\
\hline Noncausal & $\begin{array}{l}\text { Jarrod always chooses solitary activities. He requires excessive } \\
\text { attention. He is unable to remember new information. These } \\
\text { characteristics are completely separate aspects of who he is } \\
\text { (i.e., one characteristic does not cause another). }\end{array}$ & $\begin{array}{l}\text { Penny frequently suffers from insomnia. She has trouble } \\
\text { remembering the names of objects. She suffers from episodes of } \\
\text { extreme anxiety. These characteristics are completely separate } \\
\text { aspects of who she is (i.e., one characteristic does not cause another). }\end{array}$ \\
& $\begin{array}{l}\text { Because Jarrod always chooses solitary activities, he requires } \\
\text { excessive attention to make up for the lack of human contact. }\end{array}$ & $\begin{array}{l}\text { Because Penny frequently suffers from insomnia and is in a } \\
\text { habitual state of sleep deprivation, she has trouble remembering } \\
\text { the names of objects. This memory problem, in turn, leads her to }\end{array}$ \\
& $\begin{array}{l}\text { This need, in turn, causes him to be unable to remember new } \\
\text { information, because he relies on the attention of others to } \\
\text { remember all the important information for him. }\end{array}$ & $\begin{array}{l}\text { suffer from episodes of extreme anxiety, because she fears that it } \\
\text { will cause her to embarrass herself in front of others. }\end{array}$ \\
\hline
\end{tabular}


internal validity of our causal/noncausal manipulation. Twelve undergraduates rated the 30 items in the noncausal format with respect to the ease of generating a causal explanation for the features, using a 10 -point scale $(1=$ very difficult, $10=$ very easy). We excluded items with mean ratings higher than 8 .

\section{Results and Discussion}

Figure 1 summarizes the results. A 2 (causality) $\times 2$ (plausibility) mixed-design analysis of variance (ANOVA) was conducted. There was a reliable interaction between causality and plausibility $\left[F(1,69)=10.32, M S_{\mathrm{e}}=4.62\right.$, $p<.01]$. Plausible causal explanations made people appear more normal, whereas implausible causal explanations made people appear more abnormal. There was a theoretically uninteresting main effect of plausibility $\left[F(1,69)=84.23, M S_{\mathrm{e}}=37.66, p<.001\right]$, presumably because of differences in the severity of the DSM features that happened to be used for the two types of items. The main effect of causality was not reliable $[F(1,69)<1]$.

The results from the plausible causal relations condition support Meehl's (1973) prediction that understanding a person's behavior makes it seem more normal. In the situations that Meehl described, clinicians spontaneously generated causal explanations, which presumably they believed were highly plausible (otherwise they would not have generated them!). It makes sense that only plausible causal relations increase the appearance of normality, because only such explanations would be expected to increase understanding. Implausible causal relations, on the other hand, are likely to decrease understanding.

\section{EXPERIMENT 2}

Oftentimes, explanations that people interject about personality characteristics are not simply about causal relations among the characteristics but also about what precipitated these characteristics in the first place. In

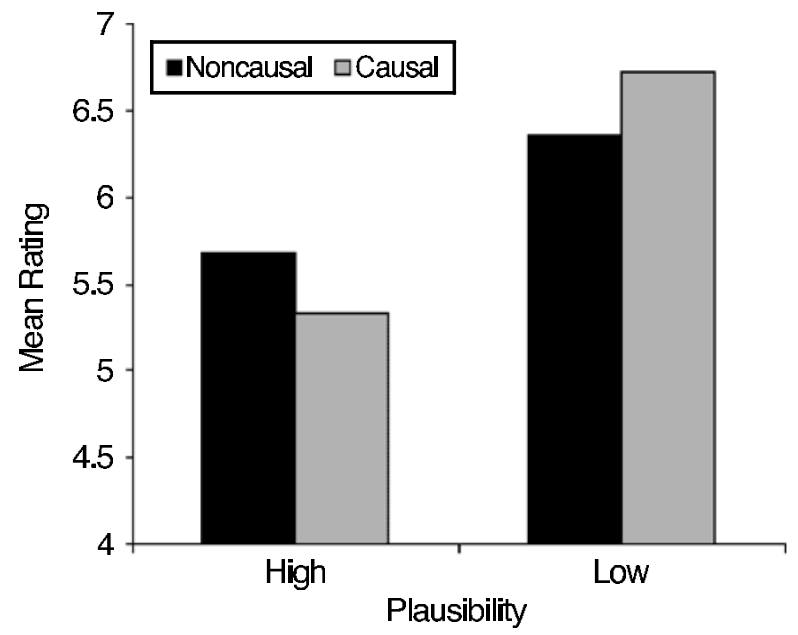

Figure 1. Mean normality ratings by undergraduate students in Experiment 1 in the causal and noncausal conditions.
Experiment 2, we examined whether these additional causal explanations would make people appear even more normal.

We considered two kinds of naive theories that people might generate to explain the mental and behavioral characteristics of others. One type of theory refers to a salient event in a person's life (e.g., "Because he was bullied a lot by his classmates when he was young, Jarrod always chooses solitary activities"), and the other refers to a brain abnormality (e.g., "Because he has a lesion in the speech center of his brain, Jarrod always chooses solitary activities"). The following two competing predictions can be made about the effects of these two types of explanations.

Previous studies in legal decision making have found that crimes attributed to internal/controllable factors (e.g., intention) lead to harsher evaluation and punishment than do crimes attributed to external/uncontrollable factors (e.g., growing up in a poor family; see, e.g., Carroll \& Payne, 1977; Grasmick \& McGill, 1994). Because less harsh punishments tend to be given when criminals are perceived as more normal, ${ }^{3}$ these results suggest that a critical determiner of normality is the external/internal distinction. Both the life event and the brain abnormality causes we used would be considered external and uncontrollable factors. Therefore, according to this view, both of these types of causes should make the person appear more normal.

However, if understanding the causal mechanism(s) underlying behavior is a key factor in making a person appear more normal, only the life event cause may further increase the perception of normality, because the mechanisms underlying the influences of life events are likely to be better understood than those underlying the influences of brain abnormalities. Indeed, a separate group of 19 undergraduates judged that (1) they had a better understanding of the causal mechanisms involving our life event explanations than those involving our brain abnormality explanations $(p<.001)$, (2) our life event explanations were more plausible than our brain abnormality explanations $(p<.05)$, and $(3)$ the life events described in our vignettes occur more frequently in real life than do the brain abnormalities $(p<.001)$.

\section{Method}

The materials for Experiment 2 were based on those used in the causal condition in Experiment 1 . In the causal control condition $(n=27)$, the materials from the Experiment 1 causal chain condition were used without change. In the life event condition $(n=28)$, the participants were additionally told how a life event caused the first characteristic in the causal chain. For instance, in the Penny example in Table 1, "Because she is very stressed out due to her workload" was added at the beginning of the causal version. In the brain abnormality condition $(n=27)$, the participants were told how a brain abnormality caused the first characteristic in the causal chain (e.g., "Because she has problems in the reticular formation of her brain stem, ...”). To emphasize that their task was to judge psychological normality rather than the normality of the life events or brain problems, we asked, "What is your first impression of this person's psychological health?" The participants answered this 
question on a 9-point scale anchored with $1=$ very good (normal) and $9=$ very poor (severely disturbed $)$.

\section{Results and Discussion}

Providing a life event explanation made people appear more normal for both the plausible and the implausible items, as is shown in Figure 2. Brain explanations did not influence normality judgments. A 3 (condition) $\times 2$ (plausibility) mixed-design ANOVA showed a reliable main effect of condition $\left[F(2,79)=4.33, M S_{\mathrm{e}}=2.04, p<\right.$ $.05]$. The brain abnormality $(M=5.94)$ and causal control $(M=5.74)$ conditions did not differ $(p>.45)$, but the items in the life cause condition $(M=5.17)$ were judged to be more normal than those in both the control and the brain abnormality conditions $[t(53)=2.17, p<.05$, and $t(53)=2.89, p<.01$, respectively]. Again, there was a theoretically uninteresting main effect of plausibility $\left[F(2,79)=121.50, M S_{\mathrm{e}}=0.309, p<.001\right]$. The interaction was marginally reliable $[F(2,79)=2.55, p<.09]$, due to a somewhat larger effect of adding the life event cause to the plausible causal chain than of adding it to the implausible causal chain.

These results support Meehl's (1973, p. 244) hypothesis that "understanding it makes it normal." Because the students thought that they understood the mechanisms underlying the psychological and behavioral consequences of the life events we used, providing such explanations increased their understanding of both the plausible and the (otherwise) implausible causal relations. In contrast, because they did not believe that they had a good understanding of the mechanisms underlying the effects of brain abnormalities, the addition of such explanations had no effect on their normality ratings. These results contradict the prediction from the attribution perspective that both the life event and the brain abnormality causes should

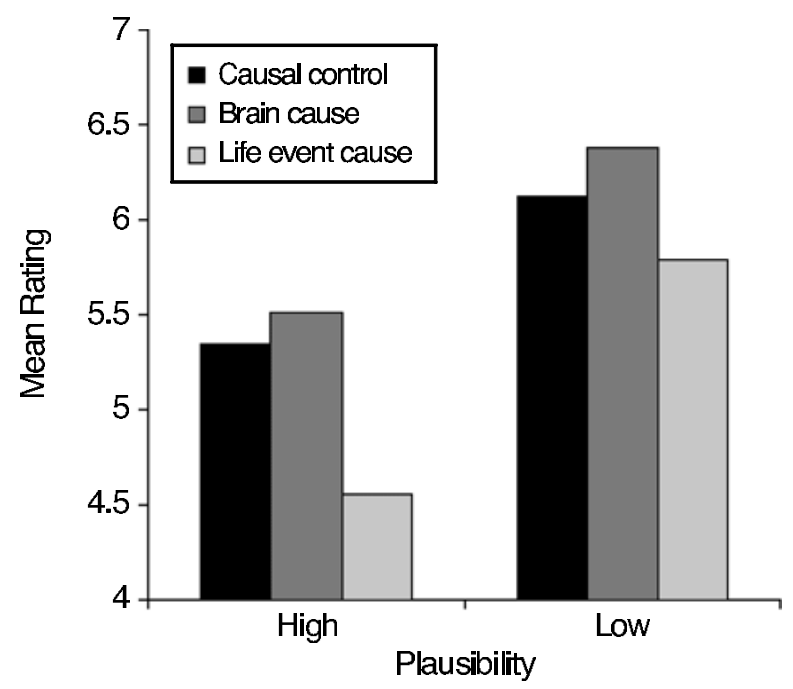

Figure 2. Mean normality ratings in Experiment 2 by undergraduate students given brain abnormality explanations, life event explanations, or no additional explanations. make people appear more normal because both types of causes are external and uncontrollable.

Increased understanding may not be the only possible cause of the perceived increase in normality in the life event condition. An alternative explanation is that adding the life event explanations made people look more normal, because the life event descriptions, although selected to be negative, implied more normal psychological health than did the mental and behavioral characteristics. To examine whether the perceived normality of the life events could predict the normality ratings from the life event condition, we asked a separate group of 30 undergraduates to rate the normality of all the characteristics (i.e., the DSM characteristics, as well as the life events and brain abnormalities), each presented as a separate characteristic of a different individual. A regression analysis was conducted to determine whether the mean ratings across the three DSM characteristics in an item and the mean ratings for the life events obtained from this group could predict the normality ratings from the life event condition. The beta weight for the $D S M$ characteristics was reliable $[\beta=$ $1.76, t(9)=3.97, p<.01]$. Contrary to the prediction made by the alternative hypothesis, the beta weight for the life events was not $(\beta=-0.13, p>.60)$. In other words, the perceived normality of the life events alone did not reliably predict normality ratings from the life event condition.

\section{EXPERIMENT 3}

Meehl's (1973, p. 244) claim that "understanding it makes it normal" was offered as a description of clinical reasoning. In our tests of this hypothesis in Experiments 1 and 2, we used undergraduate participants. In Experiment 3, we examined whether clinical psychologists also would be influenced by causal explanations when judging the normality of people.

\section{Method}

Ten experts (licensed clinical psychologists who had spent a median of 17.5 years and a range of 13-28 years seeing patients) and 10 graduate students in a clinical psychology program (3 first-year students, 2 second-year students, 3 third-year students, 1 fourthyear student, and 1 intern) were tested in the causal control and life event conditions of Experiment 2. Five participants in each expertise group were randomly assigned to each condition. Only the effect of life event explanations was examined in this experiment, because we suspected that this kind of explanation is what clinical psychologists spontaneously generate during therapy sessions.

\section{Results and Discussion}

A 2 (causal condition) $\times 2$ (plausibility) $\times 2$ (expertise) mixed-design ANOVA was conducted. The mean ratings for each condition are shown in Figure 3. There was a reliable main effect of condition $\left[F(1,16)=5.89, M S_{\mathrm{e}}=1.55\right.$, $p<.05]$, since people were judged more normal given life event explanations for their mental and behavioral characteristics than without such explanations. As in the 


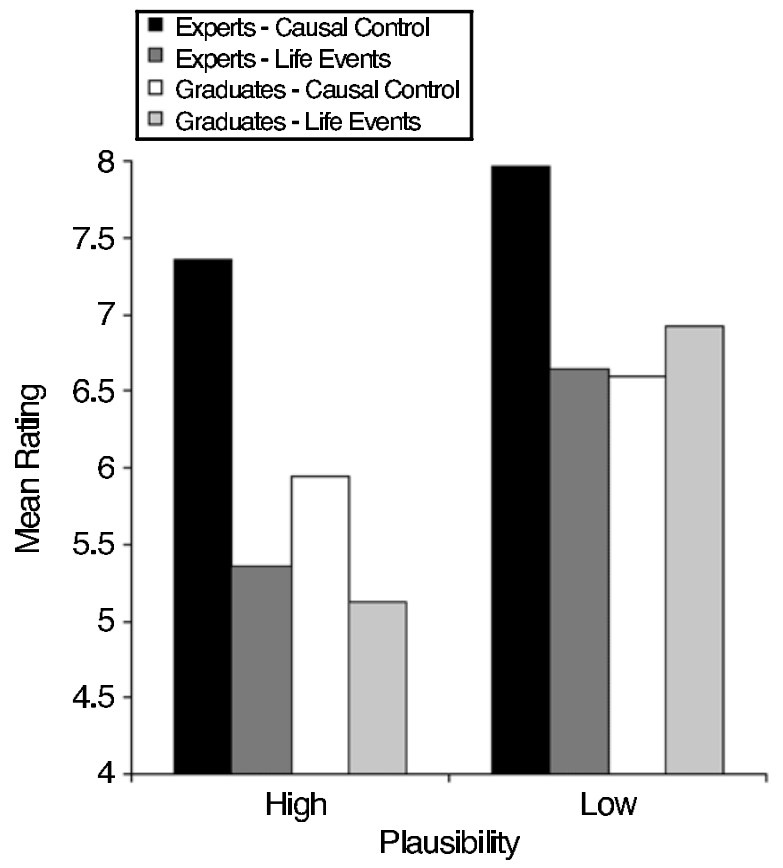

Figure 3. Mean normality ratings by experts and graduate students in clinical psychology with or without life event causes.

earlier experiments, there was a reliable main effect of plausibility $\left[F(1,16)=36.36, M S_{\mathrm{e}}=0.32, p<.001\right]$. Causal condition and plausibility interacted (replicating the pattern observed in Experiment 2), since the condition effect was more pronounced for the high-plausibility items $[F(1,16)=6.40, p<.05]$. There was also a marginally reliable main effect of expertise, since experts gave somewhat higher (more abnormal) ratings overall than did graduate students $[F(1,16)=3.03, p=.10]$. Finally, there was a marginally reliable condition $\times$ expertise interaction, because experts showed a stronger effect of our manipulation than did graduate students $[F(1,16)=$ $3.21, p=.09]$. There were no other reliable effects. These results clearly support Meehl's (1973) hypothesis; both clinical psychology experts and trainees perceived people as more normal if they had life event explanations for their mental and behavioral characteristics.

\section{GENERAL DISCUSSION}

\section{Scope of the Observed Phenomenon}

The results of the three experiments reported here provide strong evidence for the hypothesis that the presence of understandable causal relations - both plausible ones connecting one mental or behavioral characteristic to another and life event root causes for an entire causal chainmakes people seem more normal. Although these specific results are necessarily limited to the methodologicalcontext we employed and the particular type of judgment we elicited, we suspect that the phenomenon is, in fact, much broader.
With respect to the methodology, we always gave the participants specific explanations to consider, rather than letting them spontaneously generate their own explanations. In real life, people often are given causal explanations for others' behavior, both from the actors themselves and from observers. Moreover, it has been argued (e.g., Jones, 1990) that perceiving others is synonymous with making sense of their behavior by interpreting those actions in terms of intentions, causes, desires, and so forth. Therefore, it is reasonable to expect the present findings to apply as well to self-generated causal explanations.

Making sense of a person's behavior is a much more multifaceted interpretive endeavor than simply evaluating how normal or abnormal the person is. Thus, we suspect that similar findings would be obtained for other types of evaluative judgments. For instance, when the morality of another person is judged, that person might appear more moral if one can understand the reason(s) behind seemingly immoral behavior. Similarly, in an undergraduate seminar on creativity taught by one of the authors, several students spontaneously commented that many of the creative products (paintings, inventions, scientific theories) discussed in class seemed less creative (i.e., more normal) once the etiology and the method of their creation were demystified. Thus, the present phenomenon may very well reflect a general or fundamental cognitive principle.

\section{Relation to Thagard's (1989) Model of Explanatory Coherence}

Our results provide some predictive validity to Thagard's (1989) conception of the determinants of explanatory coherence. As was mentioned earlier, Thagard's model predicts that the plausible causal relations among the characteristics used in Experiment 1 would constitute a more coherent explanation of a person's behavior than would the absence of such causal relations. Also according to this model, the additional life event causes used in Experiments 2 and 3 would further increase the coherence of the explanation. To link these notions of explanatory coherence to possible consequences of having a coherent versus an incoherent explanation of someone's behavior, consider that increased coherence of an explanation seems akin to chunking. Consequently, it would seem as if the person has fewer negative characteristics-for example, there is only one thing wrong with him (likely the first characteristic in the causal chain), as opposed to three.

Recall that in Experiment 1, we found that adding implausible causal relations connecting the three characteristics made people seem less normal. Presumably, the implausible vignettes provided less coherent explanations than did the no-cause versions of those stimuli. Because of the inhibitory links in Thagard's (1989) connectionist model of explanatory coherence, it seems reasonable to suppose that the decreased coherence led 
the participants to represent these vignettes as containing more chunks than did the matched no-cause vignettes.

Given this evidence, we suggest that additional, more focused tests of the hypothesized chunking mechanism are warranted. To be persuasive, such tests will need to include independent assessments of explanatory coherence, plausibility (perhaps), chunking, and normality. Such data could clarify why the plausibility of causal relations matters, as well as why not all root cause explanations (e.g., the life events vs. brain abnormalities used in Experiment 2) necessarily have the same effect on normality judgments.

\section{On the Rationality of the Observed Phenomenon}

Meehl (1973) treated the phenomenon demonstrated in this article as a reasoning fallacy, listing it as one reason why he did not attend psychiatric case conferences where such behavior might often be observed. A clinical trainee participant in a pilot study conducted for Experiment 3 shared the same sentiment. We initially designed Experiment 3 as a within-subjects manipulation in which the participants were to rate the normality of patients described in the control condition format and then to rate them again with the life event causes revealed. This pilot study participant refused to change any of her ratings in the second round (i.e., in the life event cause condition), because she felt that it would be absurd to change her ratings of psychological normality simply because she now knew the underlying causes. In other words, when the two conditions were put before her, the fallacy of the "understanding it makes it normal" phenomenon became extremely clear.

Being free from influences of causal explanations is the philosophy underlying the $D S M-I V$ (American Psychiatric Association, 1994). The introduction to the $D S M-I V$ states that the DSM-III took "a descriptive approach that attempted to be neutral with respect to theories of etiology" (American Psychiatric Association, 1994, pp. xvii-xviii); this approach is also adopted in the $D S M-I V$. The manual represents each disorder as a checklist of symptoms, without any organizing theory to tie the symptoms together (Follette \& Houts, 1996). The $D S M$ casebook, used in training, encourages clinicians to search for symptoms in their patients that match the $D S M-I V$ (American Psychiatric Association, 1994) diagnostic criteria, without explicitly instructing them to incorporate any additional notions they may have of how these symptoms may be related (Spitzer, Gibbon, Skodol, Williams, \& First, 1994).

Determining whether the atheoretical classification system of the DSM is normative seems to depend on the definition of normative that is applied. One meaning of normative is relating to an authoritative rule or standard. Over the last 2 decades, the DSM system, which was developed by a major professional organization of clinicians, has been placed at the core of research funding, journal titles, and health care reimbursements. Thus, in practice, the DSM clearly serves as a normative base for formal diagnosis in the U.S. From this perspective, being swayed by causal explanations in judging normality is nonnormative.

A second meaning of normative involves the notion of rationality - that is, an appeal to reason, right thinking, or validity. Rather than being based on a rational principle, the atheoretical nosology of the DSM system is merely a result of a compromise arrived at to avoid battles between different theoretical schools as to which theories should be utilized in the manual. In fact, categorization based on theories represents a more sophisticated basis for categorization for which scientists should strive (Hempel, 1965). For instance, sorting animals by their evolutionary history is more sophisticated than sorting them by size. Kim and Ahn (2002) found that clinical psychologists actually do not conceptualize mental disorders in an atheoretical manner. Being sensitive to the causes of symptoms, as well as to the causal relations among symptoms, might be more rational because this information provides more useful clues for prognosis and treatment. In the case of medical diagnosis, for instance, discovering the cause of a symptom, such as nausea (e.g., bacteria vs. pregnancy), allows doctors to determine the proper course of treatment and also to make a better prognosis of the condition (e.g., will it lead to a fever or to a new baby?). In contrast, merely learning the effect of the symptom (e.g., nausea usually causes a person to throw up) does not necessarily help us to determine an appropriate treatment plan (see also Ahn $\&$ Kim, 2000, for more discussion). From this perspective, therefore, one might argue that being influenced by causal explanations in making judgments of normality is entirely rational.

\section{REFERENCES}

Ahn, W.-K., Brewer, W. F., \& Mooney, R. J. (1992). Schema acquisition from a single example. Journal of Experimental Psychology: Learning, Memory, \& Cognition, 18, 391-412.

AHN, W.-K., \& KIM, N. S. (2000). The role of causal status of features in categorization: An overview. In D. L. Medin (Ed.), Psychology of learning and motivation (Vol. 40, pp. 23-65). New York: Academic Press.

American Psychiatric Association. (1994). Diagnostic and statistical manual of mental disorders (4th ed.). Washington, DC: American Psychiatric Association.

Carroll, J. S., \& Payne, J. W. (1977). Crime seriousness, recidivism risk, and causal attributions in judgments of prison term by students and experts. Journal of Applied Psychology, 62, 595-602.

Chi, M. T., Bassok, M., Lewis, M. W., Reimann, P., \& Glaser, R. (1989). Self-explanations: How students study and use examples in learning to solve problems. Cognitive Science, 13, 145-182.

Crocker, J., Hannah, D. B., \& Weber, R. (1983). Person memory and causal attributions. Journal of Personality \& Social Psychology, 44, 55-66.

Follette, W. C., \& Houts, A. C. (1996). Models of scientific progress and the role of theory in taxonomy development: A case study of the DSM. Journal of Consulting \& Clinical Psychology, 64, 1120-1132.

Grasmick, H. G., \& McGill, A. L. (1994). Religion, attribution style, and punitiveness toward juvenile offenders. Criminology, 32, 23-46.

Hempel, C. G. (1965). Aspects of scientific explanation. New York: Free Press.

JoNES, E. E. (1990). Interpersonal perception. New York: Freeman. 
Kahneman, D., \& Tversky, A. (1982). The simulation heuristic. In D. Kahneman, P. Slovic, \& A. Tversky (Eds.), Judgment under uncertainty: Heuristics and biases (pp. 201-208). Cambridge: Cambridge University Press.

KIM, N. S., \& AHN, W.-K. (2002). Clinical psychologists' theory-based representations of mental disorders predict their diagnostic reasoning and memory. Journal of Experimental Psychology: General, 131, 451-476.

Koehler, D. J. (1991). Explanation, imagination, and confidence in judgment. Psychological Bulletin, 110, 499-519.

Meenl, P. E. (1973). Psychodiagnosis: Selected papers. Minneapolis: University of Minnesota Press.

Moore, J., Strube, M. J., \& LACKs, P. (1984). Learned helplessness: A function of attribution style and comparative performance information. Personality \& Social Psychology Bulletin, 10, 526-535.

Pennington, N., \& Hastie, R. (1992). Explaining the evidence: Tests of the story model of juror decision making. Journal of Personality \& Social Psychology, 62, 189-206.

Read, S. J., \& Marcus-Newhall, A. (1993). Explanatory coherence in social explanations: A parallel distributed processing account. Journal of Personality \& Social Psychology, 65, 429-447.

Shafir, E, Simonson, I., \& Tversky, A. (1993). Reason-based choice. Cognition, 49, 11-36.

Sherman, S. J., Zehner, K. S., Johnson, J., \& Hirt, E. R. (1983). Social explanation: The role of timing, set, and recall on subjective like- lihood estimates. Journal of Personality \& Social Psychology, 44, 1127-1143.

Spitzer, R. L., Gibbon, M., Skodol, A. E., Williams, J. B. W., \& FIRST, M. B. (EDS.) (1994). DSM-IV casebook: A learning companion to the Diagnostic and Statistical Manual of Mental Disorders, fourth edition. Washington, DC: American Psychiatric Press.

Thagard, P. (1989). Explanatory coherence. Behavioral \& Brain Sciences, 12, 435-467.

\section{NOTES}

1. The participants in Experiments 1 and 2 were undergraduates from either Yale University or Vanderbilt University, who participated in partial fulfillment of the requirements of an introductory psychology course.

2. Each description contained symptoms from three separate $D S M-I V$ disorders.

3. J. Randall LaFevor, J.D., personal communication, May 2, 2002. Mr. LaFevor has 25 years of legal experience in the state of Tennessee. $\mathrm{He}$ is a former criminal attorney, former public defender, and former juvenile court referee. He is currently an administrative law judge for the state of Tennessee.

(Manuscript received January 8, 2002; revision accepted for publication July 18 , 2002.) 